\title{
Efecto del pH en la Degradación Fotocatalítica de Materia Orgánica Natural
}

\author{
Sergio H. Valencia, Juan M. Marín, Gloria M. Restrepo \\ Universidad de Antioquia, Grupo de Procesos Fisicoquímicos Aplicados (PFA), \\ Sede de Investigación Universitaria (SIU), Carrera 53 No. 61-30, Medellín-Colombia \\ (e-mail: hvalens@gmail.com)
}

Recibido Dic. 10, 2010; Aceptado Feb. 11, 2011; Versión Final recibida Feb. 19, 2011

\section{Resumen}

Se ha estudiado el efecto del pH en la degradación fotocatalítica de materia orgánica natural del agua de un ecosistema léntico, empleando dióxido de titanio comercial en suspensión y un simulador solar UV. Para el seguimiento de la degradación y la caracterización de la materia orgánica natural se usó cromatografía de exclusión de tamaño, con un detector de carbón orgánico disuelto y un detector ultravioleta a una longitud de onda $\lambda=254 \mathrm{~nm}$, y espectrofotometría UV-Vis. Se encontró que la cinética de la reacción es alta a un pH por debajo del punto isoeléctrico del fotocatalizador y disminuye a un $\mathrm{pH}$ por encima del punto isoeléctrico. Además, la fotocatálisis se da por la alteración de la distribución de las masas moleculares, especialmente por la degradación de compuestos de alta masa molecular a compuestos de baja masa molecular, antes de su mineralización.

Palabras clave: materia orgánica natural, fotocatálisis heterogénea, dióxido de titanio, cromatografía

\section{Effect of pH on the Photocatalytic Degradation of Natural Organic Matter}

\begin{abstract}
The effect of $\mathrm{pH}$ on the photocatalytic degradation of natural organic matter in water from a lentic ecosystem using commercial titanium dioxide in suspension and a solar UV simulator was studied. The degradation monitoring and natural organic matter characterization was followed by size exclusion chromatography with dissolved organic carbon and ultraviolet detection at wave length $\lambda=254 \mathrm{~nm}$, and UV-Vis spectrophotometry. It was found that at $\mathrm{pH}$ below the isoelectric point of the photocatalyst, the kinetics of the reaction is high and with a $\mathrm{pH}$ above the isoelectric point the reaction kinetics decreases. Also, the photocatalysis is given by an alteration of the molecular weight distribution especially for the degradation of high molecular weight compounds to low molecular weight compounds, before their mineralization.
\end{abstract}

Keywords: natural organic matter, heterogeneous photocatalysis, titanium dioxide, chromatography 


\section{INTRODUCCIÓN}

La materia orgánica natural (MON) es el mayor precursor de subproductos de desinfección (DBPs), cuando se usa el cloro como desinfectante (Huang et al., 2008). El objetivo principal de los tratamientos de potabilización es remover patógenos, sustancias tóxicas y contaminantes del agua cruda. Para las aguas superficiales, incluyendo ríos, lagos y reservorios, se usa un tratamiento convencional, que incluye la coagulación, floculación, sedimentación, filtración y desinfección (Xie, 2004), pero este tratamiento convencional solo alcanza una remoción del carbono orgánico disuelto (COD) entre $50 \%$ y el $80 \%$ y lleva a que el desinfectante utilizado reaccione con la MON, para producir varios DBPs. Cerca de 600 DBPs se han identificado y entre ellos los compuestos bromados y iodados son altamente tóxicos (Krasner et al., 2006; Deborde y Gunten, 2008), los DBPs más comunes son los trihalometanos (THMs), los ácidos acéticos (HAAs), los haloacetonitrílos, las halopropanonas, las halocetonas, los clorofenoles y los aldehídos (Buchanan et al., 2006; Chang et al., 2001; Xue et al., 2008). Los THMs son un grupo de compuestos orgánicos volátiles, con formula general $\mathrm{CHX}_{3}$, donde $\mathrm{X}$ puede ser un halógeno $\mathrm{o}$ una combinación de halógenos como el $\mathrm{CHCl}_{3}, \mathrm{CHCl}_{2} \mathrm{Br}, \mathrm{CHBr}_{2} \mathrm{Cl}$ y el $\mathrm{CHBr}_{3}$ (Gallard y Gunten, 2002; Pérez et al., 2008). The international Agency for Research on cáncer (IARC) ha clasificado el cloroformo y el bromodiclorometano en el grupo B2, como probables cancerígenos para humanos y el bromoformo y el dibromoclorometano en el grupo C, como posible cancerígeno (San Juan et al., 2007).

La fotocatálisis heterogénea con $\mathrm{TiO}_{2}$, como catalizador, ha mostrado ser una técnica prometedora, para la remoción de compuestos orgánicos del agua (Huang et al., 2008). El principio de esta tecnología es la alteración de las propiedades redox de la superficie del $\mathrm{TiO}_{2}$, mediante la absorción de fotones de energía igual o superior al bandgap del semiconductor, esta alteración denominada fotoexcitación promueve reacciones de transferencia de carga, a través de la interfaz semiconductor-solución acuosa, el resultado neto es la catálisis de la reacción de oxidación de los contaminantes disueltos, por parte del semiconductor iluminado (Herrmann, 1999).

Recientemente, la fotocatálisis heterogénea ha sido reportada para la eficiente remoción de ácidos húmicos (HA) y MON (Tay et al., 2001; Li et al., 2002; Fang et al., 2005; Doll y Frimmel; 2005; Fu et al., 2006). Sin embargo, en ninguno de estos trabajos se hace un estudio del efecto del pH en la adsorción y en la degradación fotocatalítica.

\section{MATERIALES Y MÉTODOS}

\section{Reactivos}

Dióxido de titanio (P25, Degussa, Alemania), hidróxido de sodio (0.1 N, Merck), ácido clorhídrico $(0.1 \mathrm{~N}$, Merck) y filtros de membranas de acetato de celulosa, 0,45 $\mu \mathrm{m}$ (Millipore).

\section{Caracterización}

Los cambios en las características moleculares y la adsorción UV $(\lambda=254 \mathrm{~nm})$ se midieron con cromatografía de exclusión de tamaño (SEC-COD y SEC-UV ${ }_{254}$ ), la mineralización se siguió por medio de la medida del carbono orgánico disuelto (COD), usando un TOC y los cambios en la absorción UV-Vis $(\lambda=200-700 \mathrm{~nm})$ se observaron empleando un espectrofotómetro UV-Vis.

\section{Procedimiento de irradiación}

$\mathrm{El} \mathrm{TiO}_{2}$ se suspendió en $50 \mathrm{~mL}$ de agua de un lago, con $\rho(C O D)=10 \mathrm{mgL}^{-1}$ y un $\mathrm{pH}=5$, que previamente se pasó a través de filtros de membrana de celulosa de $0.45 \mu \mathrm{m}$. Para la prueba de adsorción las muestras se dejaron un tiempo determinado en la oscuridad, para alcanzar el estado de equilibrio, luego fueron irradiadas, usando un simulador solar UV (Oriel Corp., Stratford, CT), con un filtro de atenuación atmosférica (Oriel Corp., Stratford, CT), el esquema del sistema está descrito por Doll y Frimmel (2003), y finalmente las muestras se filtraron a través de membranas de celulosa de $0.45 \mu \mathrm{m}$, para separar el $\mathrm{TiO}_{2}$. 


\section{RESULTADOS Y DISCUSIÓN}

\section{Adsorción y fotólisis}

En los estudios iniciales se utilizó una $\rho\left(\mathrm{TiO}_{2}\right)=0.5 \mathrm{gL}^{-1}$, en $50 \mathrm{~mL}$ de agua del lago, a un $\mathrm{pH}=5$. El estudio de adsorción en la oscuridad mostró que inmediatamente después de la adición del $\mathrm{TiO}_{2}$ se dio una disminución del COD, donde la adsorción alcanzó el estado de equilibrio antes de cinco minutos, pero para todos los experimentos posteriores se dejó un tiempo de 30 minutos. La prueba de fotólisis, en ausencia del $\mathrm{TiO}_{2}$, no mostró cambio en la $\rho(\mathrm{COD})$, durante 270 minutos de irradiación.

\section{Efecto de la concentración de $\mathrm{TiO}_{2}$ en la degradación de la MON}

Para estudiar el efecto de $\rho\left(\mathrm{TiO}_{2}\right)$ en la degradación fotocatalítica de la MON se utilizó una $\rho\left(\mathrm{TiO}_{2}\right)$ en el rango 0.1-1.0 $\mathrm{gL}^{-1}$, a un $\mathrm{pH}$ de 5 , un tiempo de 30 minutos para alcanzar el estado de equilibrio y 180 minutos de degradación. En la Fig. 1 se observa que al aumentar $\rho\left(\mathrm{TiO}_{2}\right)$ hay un aumento de la cantidad de MON que se absorbe en la superficie del catalizador, hasta una dosis de $0.8 \mathrm{gL}^{-1}$ y un aumento en la mineralización hasta una $\rho\left(\mathrm{TiO}_{2}\right)=0.6 \mathrm{gL}^{-1}$, a partir de la cual no se observa variación en $\rho(C O D)$.

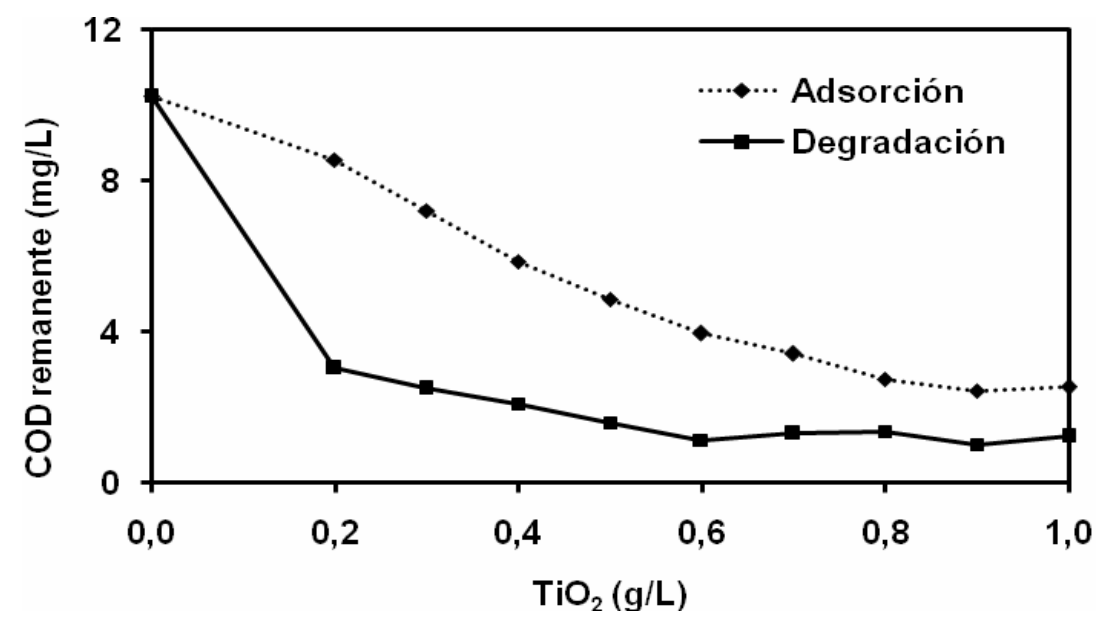

Fig. 1: Efecto de la $\rho\left(\mathrm{TiO}_{2}\right)$ en la adsorción y en la degradación de MON, $\rho_{0}(\mathrm{COD}) \approx 10 \mathrm{mgL}^{-1}$ a $\mathrm{pH}=5$, con un tiempo de adsorción 30 minutos y un tiempo de degradación 180 minutos.

La Fig. 2 muestra el perfil de degradación de la $M O N$, a un $\mathrm{pH}=5$, en función del tiempo de irradiación y de la $\rho\left(\mathrm{TiO}_{2}\right)$, durante la actividad fotocatalítica y la Fig. 3 muestra la cinética de degradación de pseudo-primer orden para la MON, donde se utilizó el modelo LangmuirHinshelwood (H-L) (Huang et al., 2008). Al aumentar $\rho\left(\mathrm{TiO}_{2}\right)$ se obtiene un aumento de $\mathrm{k}\left(\mathrm{min}^{-1}\right)$, debido al aumento del área superficial, lo que llevó al aumento de los sitios activos e incrementó la velocidad de oxidación. Sin embargo, al aumentar $\rho\left(\mathrm{TiO}_{2}\right)$ por encima de $0.8 \mathrm{gL}^{-1}$ se incrementó la turbiedad de la suspensión y redujo la penetración de la luz en el reactor, lo que disminuyó la $\mathrm{k}\left(\mathrm{min}^{-1}\right)$ (Huang et al., 2008). Por lo tanto, la dosis óptima para la degradación de la MON es 0.6 $\mathrm{gL}^{-1}$ de $\mathrm{TiO}_{2}$ a un $\mathrm{pH}=5$.

Se observa un pequeño aumento en la $\rho(\mathrm{COD})$ para $\rho\left(\mathrm{TiO}_{2}\right)$ de $0.4,0.6$ y $0.8 \mathrm{~g} / \mathrm{L}$, durante los primeros 30 minutos de irradiación, luego de la adsorción inicial, lo que se debe a la desorción, desde la superficie del $\mathrm{TiO}_{2}$, de productos intermedios de oxidación, que son más hidrofílicos que la muestra de MON original y no se adsorben fuertemente (Huang et al., 2008). 


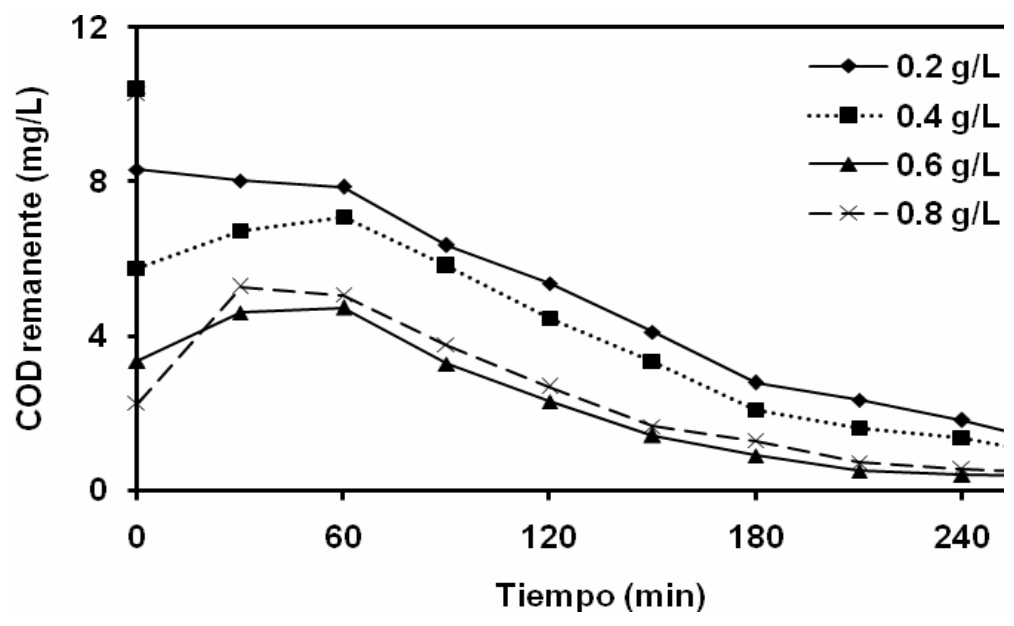

Fig. 2: Perfiles de la degradación de la MON, en función del tiempo y $\rho\left(\mathrm{TiO}_{2}\right)$, con $\rho_{0}(\mathrm{COD}) \approx 10 \mathrm{mgL}^{-1} \mathrm{a} \mathrm{pH}=5$.

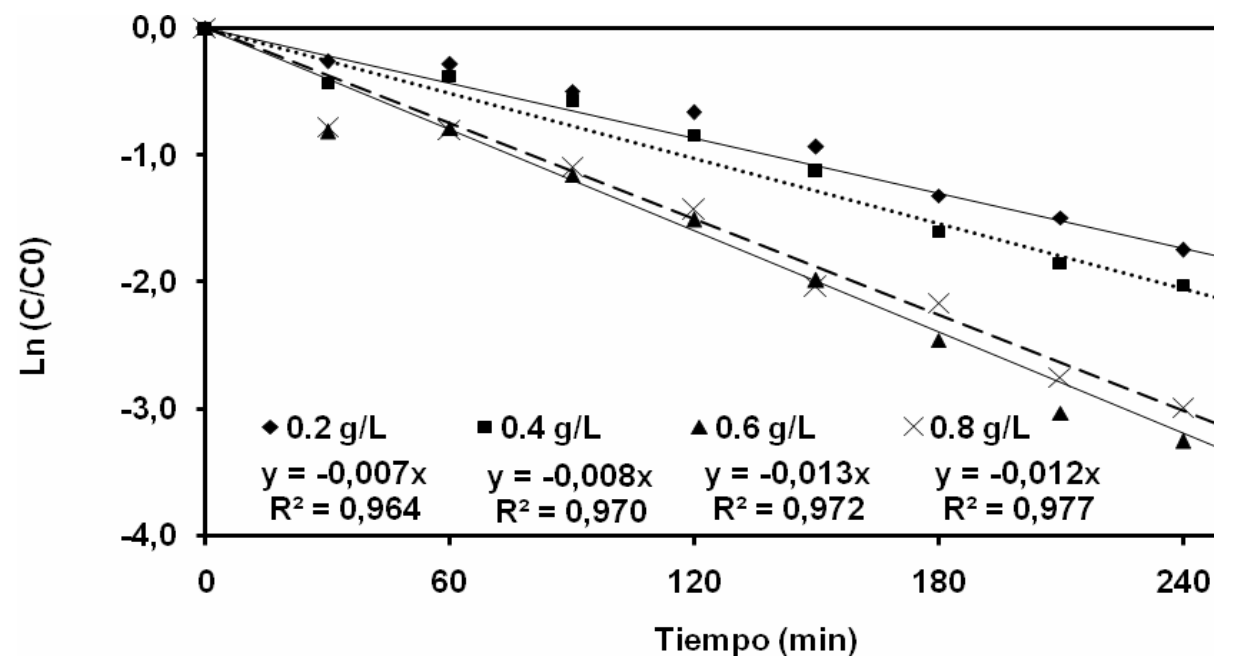

Fig. 3: Efecto de la concentración de $\mathrm{TiO}_{2}$ en la constante de degradación de pseudo-primer orden, a $\mathrm{pH}=5$.

\section{Efecto del pH en la degradación de la materia orgánica}

Para estudiar el efecto del $\mathrm{pH}$ en la degradación de la MON se analizó el efecto del pH en la adsorción de la MON en la oscuridad, en el rango de $\mathrm{pH}$ de 2 a 10, Fig. 4. Se observa que a valores de $\mathrm{pH}$ por debajo del punto isoeléctrico del $\mathrm{TiO}_{2}(\mathrm{pH}=6.5)$ hay una alta adsorción de MON y a valores de $\mathrm{pH}$ por encima del punto isoeléctrico disminuye, donde a un $\mathrm{pH}=10$ no se presenta adsorción de la MON. Por lo tanto, el $\mathrm{pH}$ es una variable a tener en cuenta en la degradación fotocatalítica de la MON.

Para estudiar el efecto del $\mathrm{pH}$ y $\rho\left(\mathrm{TiO}_{2}\right)$ en $\mathrm{k}\left(\mathrm{min}^{-1}\right)$ se trabajó con 5,6 y 7 y $0.4,0.6$ y $0.8 \mathrm{gL}^{-1}$, respectivamente, Fig. 5, donde el pH tomó estos valores debido a que son típicos en las aguas naturales. Para una $\rho\left(\mathrm{TiO}_{2}\right)$ dada, al disminuir el $\mathrm{pH}$ aumenta $\mathrm{k}\left(\mathrm{min}^{-1}\right)$, lo que se debe a que a altos valores de $\mathrm{pH}$, por encima del punto isoeléctrico del $\mathrm{TiO}_{2}(\mathrm{pH}=6.5)$, la superficie del $\mathrm{TiO}_{2}$ y MON están cargadas negativamente, lo que lleva a que se dé una repulsión y, por lo tanto, un menor valor de $\mathrm{k}\left(\mathrm{min}^{-1}\right)$. A valores de $\mathrm{pH}$ por debajo del punto isoeléctrico, el $\mathrm{TiO}_{2}$ está cargado positivamente, lo que lleva a que se dé una iteración favorable entre la superficie del $\mathrm{TiO}_{2}$ y $\mathrm{MON}$, con una fuerte adsorción de la MON y, en consecuencia, con un alto valor de $\mathrm{k}\left(\mathrm{min}^{-1}\right)$. Por lo tanto, la combinación $\rho\left(\mathrm{TiO}_{2}\right)$-pH óptimas para la degradación de la $\mathrm{MON}$ es $0.6 \mathrm{gL}^{-1}$ de $\mathrm{TiO}_{2}$ y un pH = 5. 


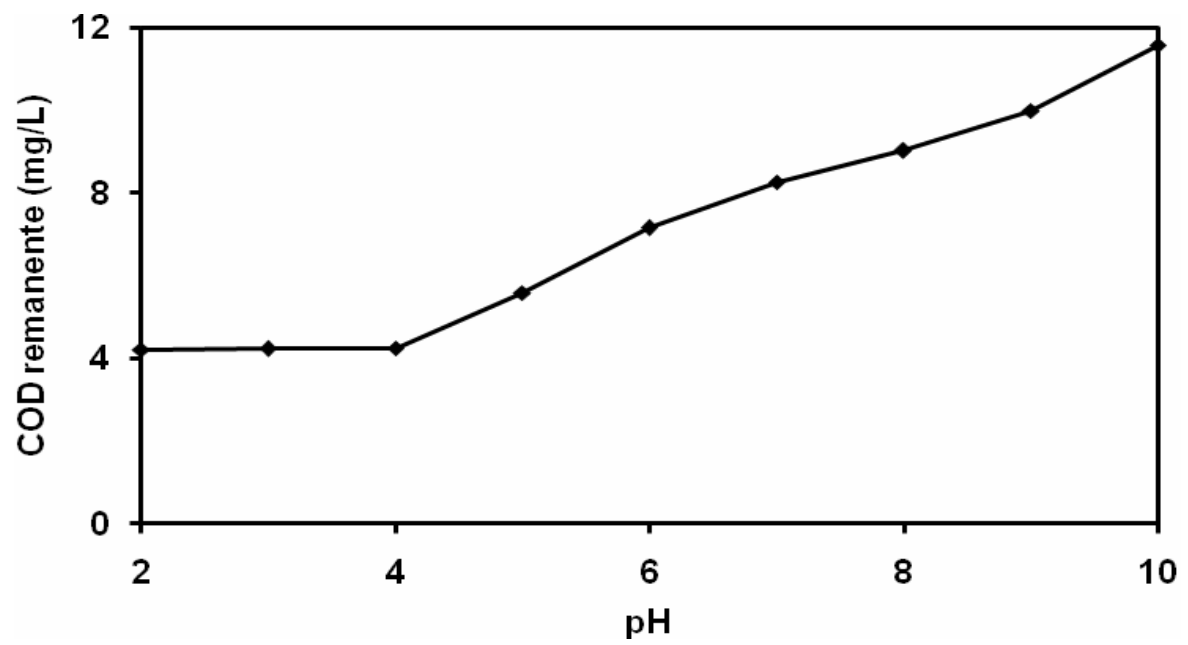

Fig. 4: Efecto del pH en la adsorción de la MON, con $\rho_{0}(C O D) \approx 10 \mathrm{mgL}^{-1} \mathrm{a} \mathrm{pH}$ $=5.0, \rho\left(\mathrm{TiO}_{2}\right)=0.6 \mathrm{gL}^{-1}$ y un tiempo de adsorción 30 minutos.

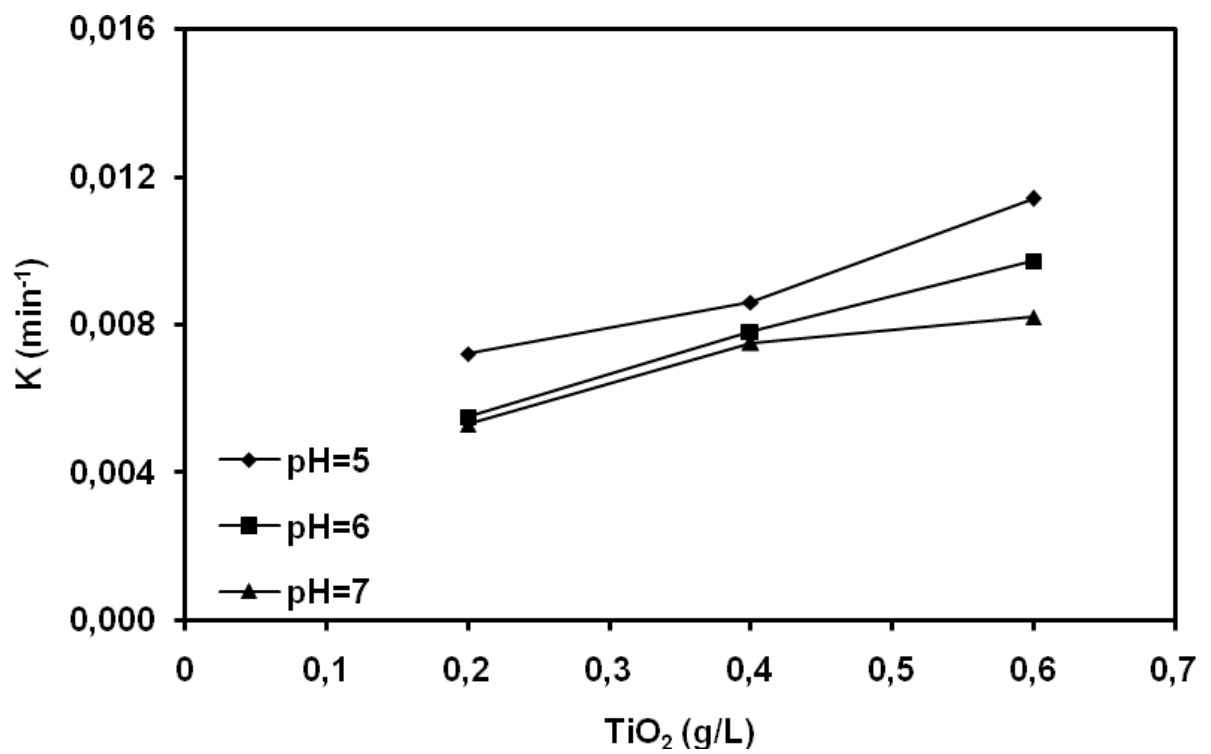

Fig. 5: Efecto de la combinación $\rho\left(\mathrm{TiO}_{2}\right)-\mathrm{pH}$ en la $\mathrm{k}\left(\mathrm{min}^{-1}\right)$ de la degradación de la MON, con $\rho_{0}(C O D) \approx 10 \mathrm{mgL}^{-1}$.

\section{Cambios en las características moleculares de la MON}

\section{Cambios en el COD}

La Fig. 6 muestra los cromatogramas SEC-COD, para las muestras antes y después de la irradiación solar UV simulada, con $\rho\left(\mathrm{TiO}_{2}\right)=0.6 \mathrm{gL}^{-1}$ y $\mathrm{pH}=5.0$, donde $\mathrm{M}_{1}$ es la muestra inicial, con $\rho_{0}(C O D)=10 \mathrm{mgL}^{-1}, \mathrm{M}_{2}$, muestra tomada luego de la adsorción, con una $\rho(C O D)=7.3 \mathrm{mgL}^{-1}$; $\mathrm{M}_{3}$, muestra tomada durante la irradiación, con $\rho(C O D)=5.9 \mathrm{mgL}^{-1} \mathrm{y} \mathrm{M}_{4}$, muestra tomada durante la irradiación, con $\rho(C O D)=3.4 \mathrm{mgL}^{-1}$. 


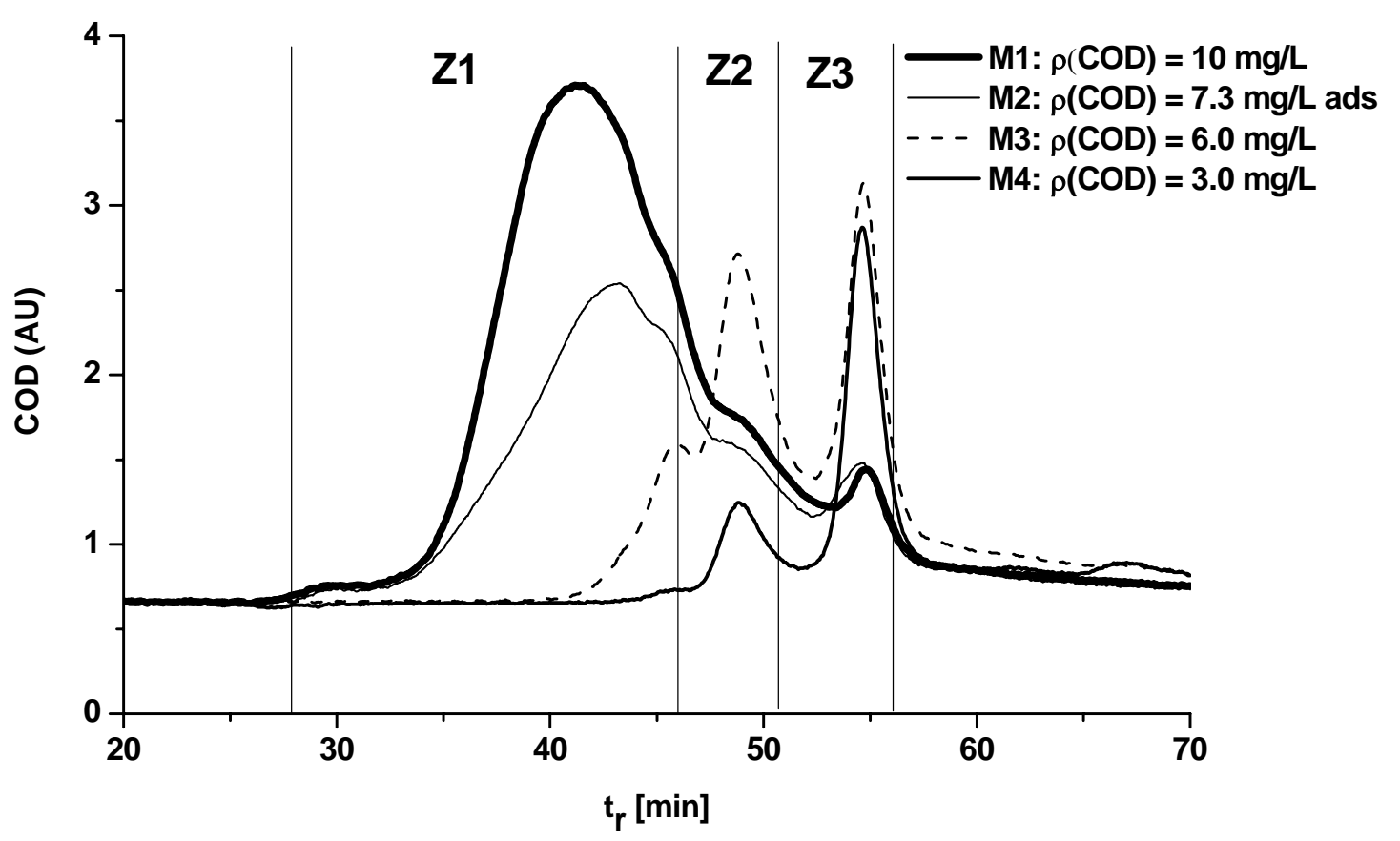

Fig. 6: Cromatograma SEC-COD para la $\mathrm{MON}$, con $\rho\left(\mathrm{TiO}_{2}\right)=0.2 \mathrm{gL}^{-1}$ a $\mathrm{pH}=5 . \mathrm{M} 1$ : $\rho_{0}(C O D) \approx 10.0 \mathrm{mgL}^{-1}, \mathrm{M} 2: \rho(C O D)=7.3 \mathrm{mgL}^{-1}, \mathrm{M} 3: \rho(C O D)=5.9 \mathrm{mgL}^{-1}, \mathrm{M} 4:$ $\rho(C O D)=3.4 \mathrm{mgL}^{-1}$.

Estos cromatogramas se dividieron en tres zonas para el análisis: la zona $1\left(Z_{1}\right)$ comprendida en el rango $28.0 \mathrm{~mL}<V_{R}<45.8 \mathrm{~mL}\left(V_{R}\right.$ es igual al tiempo de elución de 1 minuto), donde se encuentran dos picos característicos, el más alto a $V_{R}=41,9 \mathrm{~mL}$ y el más corto (un hombro) a $V_{R}$ $=43.5 \mathrm{~mL}$, estos picos se atribuyen a material húmico (Huber et al., 1994); la zona $2\left(Z_{2}\right)$ comprendida en el rango $45.8 \mathrm{~mL}<\mathrm{V}_{\mathrm{R}}<50.7 \mathrm{~mL}$, que presenta un pico característico a $\mathrm{V}_{\mathrm{R}}=46.8$ $\mathrm{mL}$, el COD de esta zona se debe a ácidos orgánicos aromáticos y polifuncionales (Huber et al., 1994, Frimmel eta., 1998); la zona $3\left(Z_{3}\right)$ comprendida en el rango $50.7 \mathrm{~mL}<V_{R}<56.5 \mathrm{~mL}$, con un pico máximo a $V_{R}=52.7 \mathrm{~mL}$, esta zona incluye moléculas que eluyen prematuramente, debido a diferencias de fuerzas iónicas, entre la fase móvil y la muestra (Huber et al., 1994; Frimmel, 1998; Tercero et al., 2009).

Durante la adsorción en la oscuridad, se observa que $Z_{1}$ se adsorbe más extensamente que $Z_{2}$ y $Z_{3}$. Por lo tanto, hay una adsorción preferencial de las fracciones de mayor peso molecular, como lo reportaron Tercero et al. (2009) y Vermeer y Koopal (1998); después de la irradiación, cuando se pasa de $\rho(C O D)=10 \mathrm{mgL}^{-1}$ a $\rho(C O D)=6.0 \mathrm{mgL}^{-1}$, se observa una rápida reducción de $Z_{1}$ y un incremento de $Z_{2}$ y $Z_{3}$, donde la reducción de $\rho(C O D)$ de $Z_{1}$ se da por la pérdida de la porción más grande. Además, el pico más alto en $Z_{1}$ cambio a dos picos y en $Z_{2}$ y $Z_{3}$ hay un aumento de los picos característicos y cuando se llega a $\rho(C O D)=3.0 \mathrm{mgL}^{-1}$ se observa una reducción total del pico de $Z_{1}$ y una reducción del pico de $Z_{2}$ y $Z_{3}$.

Se observa que la degradación de la MON no lleva inmediatamente a la mineralización, sino a la formación de compuestos más pequeños en $Z_{2}$ y $Z_{3}$, esto es, $Z_{2}$ crece a medida que $Z_{1}$ se reduce, pero llega a un máximo y declina cuando $Z_{1}$ es mínimo, esto también se da para $Z_{3}$. Por lo tanto, la degradación de la MON se da por alteración de la distribución de los tamaños moleculares, con una degradación preferencial de compuestos de alto peso molecular.

Según Tercero et al. (2009) las reacciones redox fotoiniciadas llevan a que la degradación de la MON ocurra en la superficie o en la vecindad del $\mathrm{TiO}_{2}$, lo que conduce a que $Z_{1}$ sea preferiblemente degradada que $Z_{2}$ y $Z_{3}$, a medida que ocupa una mayor superficie del $\mathrm{TiO}_{2}$ y que este fenómeno no solo ocurre en la etapa inicial, donde la MON pre-adsorbida predomina, sino también en las últimas etapas, donde el transporte y la adsorción desde la solución aportan la MON para la degradación, de tal forma que $Z_{1}$ se siga adsorbiendo preferiblemente, más que $Z 2$ y 
Z3 o los productos de degradación, y concluye que las diferencias en el comportamiento de la adsorción de las fracciones introduce selectividad de la fotocatálisis. Lo anterior se cumple cuando se tienen $\mathrm{pH}$ bajos, donde se da una fuerte adsorción de la MON en la oscuridad, pero para un $\mathrm{pH}$ $=10$, donde no ocurre la adsorción de la MON en la oscuridad, Fig. 4, y se da la degradación, Fig. 7, el proceso no se debe basar en la adsorción de la MON en la oscuridad, esto se podría explicar teniendo en cuenta el fenómeno de fotoadsorción (Serpone y Emeline, 2002; Yurdakal et al., 2009; Augugliaro et al., 2010), donde las propiedades de la superficie del $\mathrm{TiO}_{2}$ cambian bajo irradiación, lo que lleva a que se dé la adsorción de la MON, bajo irradiación o también se puede explicar por un método indirecto de degradación (Monllor-Satoca et al., 2007). Por lo tanto, el pH influye tanto en la adsorción como en la velocidad de degradación de la MON.

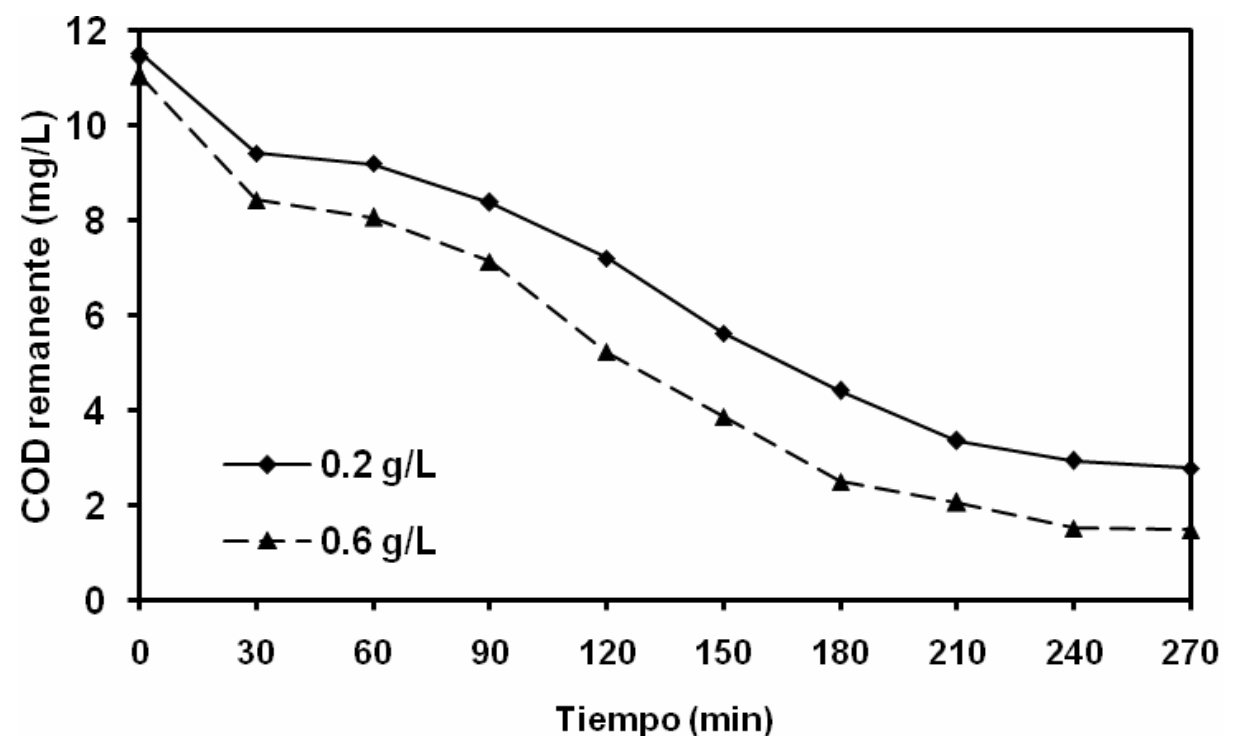

Fig. 7: Efecto del tiempo en la degradación de la $\mathrm{MON}$ a diferentes $\rho\left(\mathrm{TiO}_{2}\right)$, con $\rho_{0}(\mathrm{COD}) \approx 10 \mathrm{mgL}^{-1} \mathrm{a} \mathrm{pH}=10$.

\section{Cambios en la absorbancia UV/Vis y $U V_{254}$}

La Fig. 8 muestra la absorbancia UV/Vis de la MON, en el rango de $200 \mathrm{~nm}$ a $750 \mathrm{~nm}$, durante la degradación fotocatalítica, con $\rho\left(\mathrm{TiO}_{2}\right)=0.6 \mathrm{gL}^{-1}$ y $\rho(\mathrm{COD})=10 \mathrm{mgL}^{-1}$ a $\mathrm{pH}=5$. En la adsorción de la MON, en la superficie del $\mathrm{TiO}_{2}$, a $\mathrm{pH}=5$, se observa un alto decrecimiento de la UV/Vis y durante la irradiación hay una disminución continua de la UV/Vis, hasta un tiempo de irradiación de 120 minutos, donde a partir de este tiempo la velocidad de remoción se hace muy lenta, lo que determina que se han alcanzado MON de difícil degradación.

La Fig. 9 muestra los cromatogramas SEC-UV 254 , para las muestras antes y después de la irradiación solar UV simulada, con $\rho\left(\mathrm{TiO}_{2}\right)=0.6 \mathrm{gL}^{-1}$ a pH $=5.0$. Los cromatogramas SEC-UV 254 son cualitativamente similares a los SEC-COD, pero la velocidad de remoción de $U_{254}$ es más rápida que la remoción de COD. Los cromatogramas $S E C-U V_{254}$ también se dividieron en tres zonas, como en los SEC-COD, donde $Z_{1}$ tiene un pico característico a $V_{R}=29 \mathrm{~mL}$ y en $Z_{2}$ y $Z_{3}$ los picos son más pequeños comparados con los SEC-COD. Se observa que la $U_{2} V_{254}$, que es un indicador de la aromaticidad e hidrofobicidad, disminuye, de tal forma que a medida que ocurre la fotodegradación hay una disminución de $Z_{1}$ y un pequeño aumento de $Z_{2}$ y $Z_{3}$, cuando se pasa de $\rho(C O D)=10 \mathrm{mgL}^{-1}$ a $\rho(C O D)=6.0 \mathrm{mgL}^{-1}$ y luego se da una disminución de $Z_{1}, Z_{2}$ y $Z_{3}$, al alcanzar $\rho(C O D)=3.0 \mathrm{mgL}^{-1}$. Por lo tanto, la fotocatálisis de la MON se da por la adsorción y la degradación preferencial de compuestos que absorben altamente $U_{254}$ a compuestos con baja absorción UV 254 . 


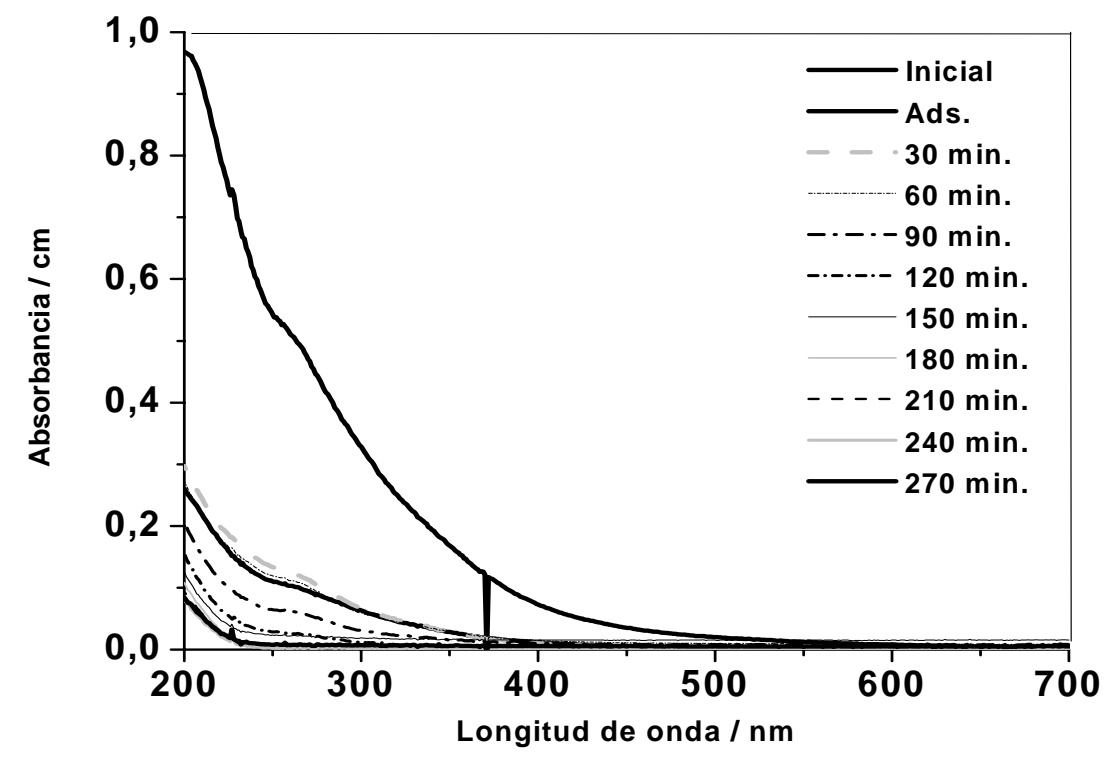

Fig. 8: Espectros UV/Vis de la MON a diferentes tiempos de irradiación con $\rho_{0}(\mathrm{COD}) \approx 10 \mathrm{mgL}^{-1}$ a $\mathrm{pH}=5$ y $\rho\left(\mathrm{TiO}_{2}\right)=0.6 \mathrm{gL}^{-1}$.

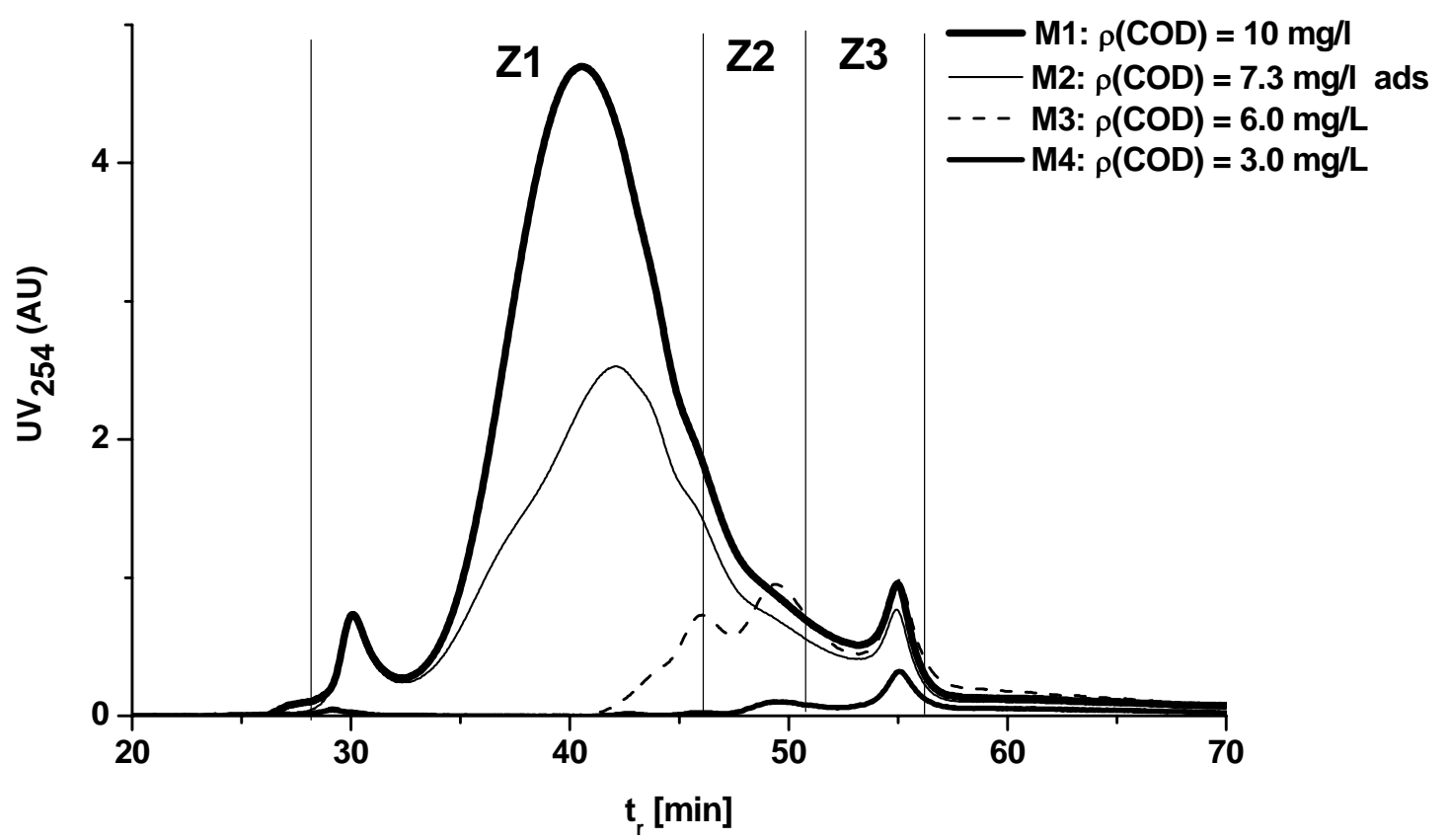

Fig. 9: Cromatograma SEC-UV $V_{24}$ para la $\mathrm{MON}$, con $\rho\left(\mathrm{TiO}_{2}\right)=0.2 \mathrm{gL}^{-1}$ a $\mathrm{pH}=5$. $\mathrm{M} 1$ : $\rho(C O D) \approx 10.0 \mathrm{mgL}^{-1}, \mathrm{M} 2: \rho(\mathrm{COD})=7.0 \mathrm{mgL}^{-1}, \mathrm{M} 3: \rho(\mathrm{COD})=6.0 \mathrm{mgL}^{-1}, \mathrm{M} 4: \rho(\mathrm{COD})=$ $3.0 \mathrm{mgL}^{-1}$.

\section{CONCLUSIONES}

La degradación fotocatalítica de la MON depende de la dosis de $\mathrm{TiO}_{2}$ y del $\mathrm{pH}$, donde a bajos valores de $\mathrm{pH}$, lejos del punto isoeléctrico del $\mathrm{TiO}_{2}$, se obtienen mayores velocidades de degradación. Además, el pH también influye en la adsorción de la MON en la oscuridad, a bajos valores de $\mathrm{pH}$ la degradación se da por la alta adsorción de la $\mathrm{MON}$, en la superficie del $\mathrm{TiO}_{2}$, donde hay una preferible adsorción de las moléculas de alto peso molecular, con alta absorción $U V_{254}$ y son degradadas a moléculas de bajo peso molecular y baja absorción $U_{254}$, lo que lleva a que se tenga una selectividad en la degradación fotocatalítica de la $\mathrm{MON}$ y a un $\mathrm{pH}$ de 10 , donde 
no hay adsorción de la MON, en la oscuridad, la degradación se puede dar debido al fenómeno de fotoadsorción o puede darse un mecanismo indirecto de degradación fotocatalítica.

\section{AGRADECIMIENTOS}

Los autores agradecen al Programa de investigación Expedición Antioquia 2013 por la Financiación del Proyecto "Procesos Innovadores para la Potabilización de Aguas en Comunidades Rurales Antioqueñas", a la Universidad de Antioquia y al Departamento Administrativo de Ciencia, Tecnología e Innovación COLCIENCIAS.

\section{REFERENCIAS}

Augugliaro, V., V. Loddo, M. Pagliaro, G. Palmisano y L. Palmisano, Clean by light irradiation, practical applications of supported $\mathrm{TiO}_{2}, 1^{\text {a }}$ edición, The Royal Society of Chemistry, Cambridge, Inglaterra (2010).

Buchanan, W., F. Roddick y N. Porter, Formation of hazardous by-products resulting from the irradiation of natural organic matter: Comparison between UV and VUV irradiation, Chemosphere: 63(7), 1130-141 (2006).

Chang, E., Y. Lin. y P. Chiang, Effects of bromide on the formation of THMs and HAAs, Chemosphere: 43(8), 1029-1034 (2001).

Deborde, M. y V. Gunten, Reaction of chlorine with inorganic and organic compounds during water treatment-Kinetics and mechanisms: A critical review, Water research: 42(1-2), 13-51 (2008).

Doll, T. y F.H. Frimmel, Fate pharmaceuticals-photodegradation by simulated solar UV-light, Chemosphere: 52(10), 1757-1769 (2003).

Doll, T. y F.H. Frimmel, Photocatalytic degradation of carbamazepine, clofibric acid and iomeprol with P25 and Hombikat UV100 in presence of natural organic matter (NOM) and other organic water constituents, Water Research: 39(2-3), 403-411 (2005).

Fang, H., D.D. Sun, M. Wu, W. Phay y J.H. Tay, Removal of humic acid foulant from ultrafiltration membrane surface using photocatalytic oxidation process, Water science and Technology: 51(6-7), 373-380 (2005).

Frimmel, F.H., Characterization of natural organic matter as major constituents in aquatic systems, Journal of Contaminant Hydrology: 35(1-3), 201-216 (1998).

Fu, J., M. Ji, Z. Wang, L. Jin y D. An, A new submerged membrane photocatalysis reactor (SMPR) for fulvic acid removal using a nano-structured photocatalyst, Journal of Hazard Materials B: 131(13), 238-242 (2006).

Gallard, H. y U. Gunten, Chlorination of natural organic matter: Kinetics of chlorination and of THMS formation, Water Research: 36(1), 65-74 (2002).

Herrmann, J., Heterogeneous photocatalysis: fundamentals and applications to the removal of various types of aqueous pollutants, Catalysis Today: 53(1), 115-129 (1999).

Huang, X., M. Leal, y Q. Li, Degradation of natural organic matter by $\mathrm{TiO}_{2}$ photocatalytic oxidation and its effect on fouling of low-pressure membranes, Water Research: 42(4-5), 1142-1150 (2008).

Huber, S.A., A. Balz y F. H. Frimmel, Identification of diffuse and point sources of dissolved organic carbon(DOC) in a small stream (Alb, southwest German), using gel filtration chromatography with high sensitivity DOC-detection, Fresenius Journal of Analytical Chemistry: 350(7-9), 496-503 (1994). 
Krasner, S., H. Weinberg, S. Richardson, S. Pastor, R. Chin, M. Sclimenti, G. Onstand y A. Thruston, Ocurrence of a new generation of disinfection byproducts, Environmental Science and Technology: 40(23), 7175-7185 (2006).

$\mathrm{Li}, \mathrm{X} . Z$., C.M. Fan y Y.P. Sun, Enhancement of photocatalytic oxidation of humic acid in $\mathrm{TiO}_{2}$ suspensions by increasing cation strength, Chemosphere: 48(4), 453-460 (2002).

Monllor-Satoca, D., R. Gómez, M. González-Hidalgo y P. Salvador, The "Direct-Indirect" model: An Alternative approach in heterogeneous photocatalysis based on the degree of interaction of dissolved pollutant species with the semiconductor surface, Catalysis Today: 129(1-2), 247-255 (2007).

Pérez, J., S. Herrero y C. Garcia, Determination of trihalomethanes in water samples: a review, Analytical Chemical Act: 629(1-2), 6-23 (2008).

San Juan, P., J. Carrillo y M. Tena, Fibre selection base on an overall analytical feature comparison for the solid-phase microextraction of trihalomethanes from drinking water, Journal of Chromatography A: 1139(1), 27-35 (2007).

Serpone, N. y A.V. Emeline, Suggested terms and definitions in photocatalysis and radiocatalysis, International Journal of Photoeenrgy: 4(3), 91-131 (2002).

Tay, J.H., D. Chen y D.D. Sun, Removal of color substances using photocatalytic oxidation for membrane filtration processes, Water Science and Technology: 43(10), 319-325 (2001).

Tercero, L., E. Haseborg, M. Weber y F. Frimmel, Investigation of the photocatalytic degradation of brown water natural organic matter by size exclusion chromatography, Applied Catalysis B: Environmental: 87(1-2), 56-62 (2009).

Vermeer, A. y L. Koopal, Adsorption of Humic Acids to Mineral Particles. 2. Polydispersity Effects with Polyelectrolyte Adsorption, Langmuir: 14(15), 4210-4216 (1998).

Xie, Y.F, Disinfection byproducts in drinking water: Formation, analysis, and control, $1^{\text {a }}$ edición, 12. Lewis Publishers, Florida, US (2004).

Xue, S., Q. Zhao, L. Wei y T. Jia, Trihalomethane formation potential of organic fractions in secondary effluent, Journal of Environmental Sciences: 20(5), 520-527 (2008).

Yurdakal, S., V. Loddo, G. Palmisano, V. Augugliaro y L. Palmisano, A quantitative method of photoadsorption determination for irradiated catalyst in liquid-solid system, Catalysis Today: 143 (1-3), 189-194 (2009). 